\title{
Regulation of caspase-3 processing by clAP2 controls the switch between pro-inflammatory activation and cell death in microglia
}

\author{
E Kavanagh ${ }^{1,3}$, J Rodhe ${ }^{1,3}$, MA Burguillos ${ }^{1}$, JL Venero ${ }^{2}$ and B Joseph ${ }^{\star, 1}$
}

The activation of microglia, resident immune cells of the central nervous system, and inflammation-mediated neurotoxicity are typical features of neurodegenerative diseases, for example, Alzheimer's and Parkinson's diseases. An unexpected role of caspase-3, commonly known to have executioner role for apoptosis, was uncovered in the microglia activation process. A central question emerging from this finding is what prevents caspase-3 during the microglia activation from killing those cells? Caspase-3 activation occurs as a two-step process, where the zymogen is first cleaved by upstream caspases, such as caspase-8, to form intermediate, yet still active, p19/p12 complex; thereafter, autocatalytic processing generates the fully mature p17/p12 form of the enzyme. Here, we show that the induction of cellular inhibitor of apoptosis protein 2 (cIAP2) expression upon microglia activation prevents the conversion of caspase-3 319 subunit to p17 subunit and is responsible for restraining caspase- 3 in terms of activity and subcellular localization. We demonstrate that counteracting the repressive effect of cIAP2 on caspase-3 activation, using small interfering RNA targeting CIAP2 or a SMAC mimetic such as the BV6 compound, reduced the pro-inflammatory activation of microglia cells and promoted their death. We propose that the different caspase-3 functions in microglia, and potentially other cell types, reside in the active caspase-3 complexes formed. These results also could indicate cIAP2 as a possible therapeutic target to modulate microglia pro-inflammatory activation and associated neurotoxicity observed in neurodegenerative disorders.

Cell Death and Disease (2014) 5, e1565; doi:10.1038/cddis.2014.514; published online 11 December 2014

\section{Introduction}

Microglia cells are the resident immune cells of the central nervous system, constantly screening the brain environment. They express surface receptors to detect changes in their environment due to brain damage or infections. An important family of these sensors is the toll-like receptor (TLR) family. ${ }^{1}$ Although microglia are necessary for normal function, uncontrolled and over-activated microglia can result in disastrous neurotoxic consequences. Indeed, microglia are a predominant source of pro-inflammatory mediators including cytokines, complement factors, free radicals, nitric oxide (NO), chemokines and prostanglandins, all of which potentially contribute to further neuronal dysfunction and death. ${ }^{1-3}$ Activation of microglia towards a pro-inflammatory phenotype and the resulting inflammatory response are typical features of neurodegenerative and neuroinflammatory disorders and have an important role in the demise of different neuronal populations. In fact, evidence from numerous clinical neuropathological observations and in vivo studies suggest a prominent role of activated microglia in the initiation and/or aggravation of neurodegenerative disorders, including Alzheimer's disease $(A D)$ and Parkinson's disease (PD). ${ }^{1,3-5}$
Caspases, a family of cysteinyl aspartate-specific proteases, are best known as executioners of apoptotic cell death and their activation are considered to be a commitment to cell death. ${ }^{6}$ However, certain caspases also function as regulatory molecules for immunity, cell differentiation and cell fate determination. We have characterized a novel and unexpected mechanism involved in the activation of microglia in response to different TLR4 ligands. This mechanism involves a caspase-dependent signaling governing microglia activation. We showed that the orderly activation of caspase-8 and caspase-3 (so-called apoptotic caspases) regulate microglia activation via a protein kinase $\mathrm{C}(\mathrm{PKC} \delta)$-dependent pathway. We found that the stimulation of microglia with various TLR ligands activates caspase- 8 and caspase- 3 in microglia without triggering cell death in vitro and in vivo. Knockdown or chemical inhibition of each of these caspases hindered microglia activation. Moreover, we provided compelling evidence that these caspases are activated in microglia in the ventral mesencephalon of PD and the frontal cortex of $A D$ subjects. $^{5}$

One central question emerging from the discovery that the apoptotic caspases regulate microglia activation is, which

\footnotetext{
${ }^{1}$ Department of Oncology-Pathology, Cancer Centrum Karolinska, R8:03, Karolinska Institutet, Stockholm, Sweden and ${ }^{2}$ Departamento de Bioquímica y Biología Molecular, Facultad de Farmacia, Universidad de Sevilla and Instituto de Biomedicina de Sevilla (IBiS), Sevilla, Spain

${ }^{*}$ Corresponding author: B Joseph, Department of Oncology-Pathology,Cancer Centrum Karolinska, R8:03, Karolinska Institutet, 17176 Stockholm, Sweden. Tel: +46 (0) 8 517738 26; Fax: +46 (0) 83390 31; E-mail: bertrand.joseph@ki.se

${ }^{3}$ These authors contributed equally to this work.

Abbreviations: AD, Alzheimer's disease; BIR, baculovirus IAP repeat; CIAP, cellular inhibitor of apoptosis protein; IAP, inhibitor of apoptosis protein; IBM, IAPbinding motif; LPS, lipopolysaccharide; NO, nitric oxide; PARP, poly(ADP-ribose) polymerase; PD, Parkinson's disease; PKC, protein kinase C; siRNA, small interfering RNA; SMAC, second mitochondria-derived activator of caspases; STS, staurosporine; TLR, toll-like receptor; XIAP, X-linked IAP

Received 17.7.14; revised 13.10.14; accepted 28.10.14; Edited by A Verkhratsky
} 
mechanism prevents them from killing the microglia cells? In this study, we show that the control of caspase-3 cleavage by cellular inhibitor of apoptosis protein 2 (clAP2) dictates whether microglia will undergo pro-inflammatory activation or apoptosis.

\section{Results}

Distinctive caspase-3 processing profile in microglia cells after pro-inflammatory stimulus. In an attempt to characterize the molecular events that hinder active caspase-3 from killing microglia, a biochemical analysis of caspase-3 processing was performed in activated microglia cells versus dying microglia cells. Caspase- 3 is synthesized as a singlechain inactive zymogen, containing a prodomain, as well as large and small subunits that include the residues required for substrate recognition and cleavage. Caspase-3 activation occurs in two stages. ${ }^{7}$ First, caspase- 3 proforms are cleaved by upstream caspases, such as active caspase-8, at Asp175 to generate intermediate, yet still active, heterotetramer complexes consisting of two p19 and two p12 peptides (p19/p12 complexes). The second stage involves removal of the short prodomain from the $\mathrm{p} 19$ peptides by autocatalytic processing, and cleavage at residue Asp28, to generate the fully mature p17/p12 form of the enzyme (see scheme in Figure 6). BV2 microglia cells were stimulated with lipopolysaccharide (LPS), the major component of Gram-negative bacterial walls and a ligand for TLR4, to investigate the processing of caspase-3 in activated microglia. Of note, intracerebral delivery of LPS, which leads in vivo to microglia activation and neuronal injury, is used as model for brain

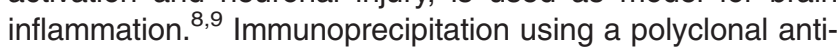
body raised against cleaved caspase-3 Asp175, which recognized both $\mathrm{p} 17$ and $\mathrm{p} 19$ subunit, was used to isolate and concentrate caspase- 3 subunits. Subsequent immunoblot analysis using the same antibody revealed that upon LPS-induced microglia activation, the processing of the p19 $\mathrm{N}$-terminal caspase-3 fragment containing the prodomain to the active p17 fragment is prevented (Figure 1a). In contrast to LPS treatment, exposure of BV2 microglia cells to a death stimulus such as staurosporine (STS) led to a significantly greater caspase-3 processing and appearance of the active p17 fragment (Figure 1a). This results are in agreement with the reported moderate $\mathrm{D}(\mathrm{OMe}) \mathrm{E}(\mathrm{OMe}) \mathrm{VD}(\mathrm{OMe})$-ase (DEVDase) activity, which reflects caspase-3 function as protease, observed upon treatment of BV2 microglia cells with various pro-inflammogens, including LPS $^{5}$ (Figure 3f).

Cytoplasmic retention of active caspase-3 in proinflammatory stimulated microglia cells. We hypothesized that during microglia activation, the avoidance of p19 to p17 caspase-3 conversion controls both the strength of activity and the localization of caspase-3. It is worth noting that nuclear localization of active caspase- 3 appears to be regulated by an active transport system. ${ }^{10,11}$ In addition, removal of the short prodomain from caspase-3 fragments (i.e., conversion of $\mathrm{p} 19$ to $\mathrm{p} 17$ ) contributes to its nuclear relocalization. ${ }^{12}$ We previously reported using confocal cell imaging that following LPS treatment of microglia BV2 cells, a

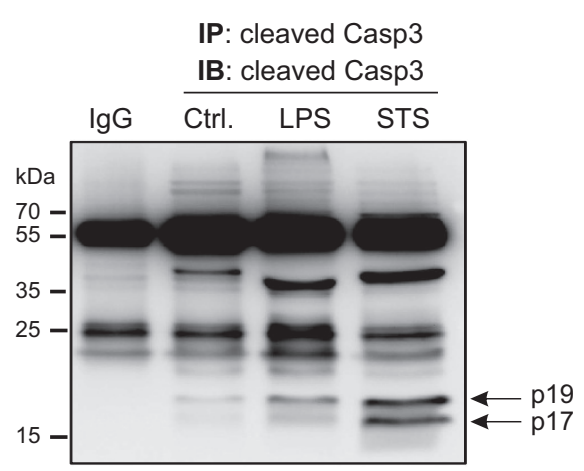

b

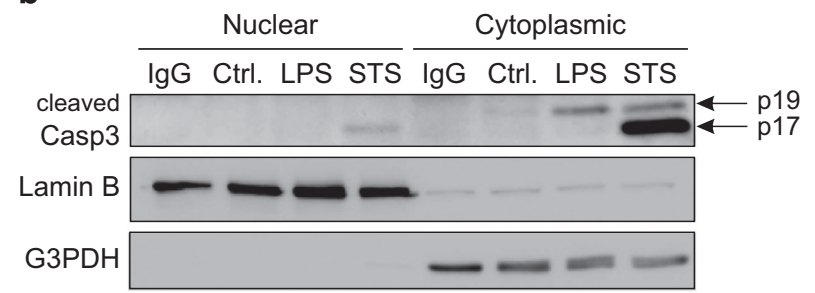

Figure 1 Distinctive caspase-3 processing profile in pro-inflammatory activated versus dying microglia. BV2 microglia cells were treated with $1 \mu \mathrm{g} / \mathrm{ml} \mathrm{LPS} \mathrm{for} 24 \mathrm{~h}$ or $0.1 \mu \mathrm{M} \mathrm{STS}$ for $3 \mathrm{~h}$ to promote pro-inflammatory activation or cell death, respectively. (a) Immunoblot analysis demonstrates presence of the 19 caspase-3 subunit in the immune complexes formed after pull down with cleaved caspase-3 Asp175 antibody, which recognizes both $\mathrm{p} 17$ and p19 subunits, in LPS-treated microglia. Immune complexes from STS-treated microglia contained both p17 and p19 caspase-3 subunits. (b) Subcellular fractionation illustrates the restricted cytoplasmic localization of p19 subunit, in contrast to the p17 subunit that was found to localize in both the cytoplasmic and the nuclear fractions

cleaved caspase-3 Asp175 is found to be located outside the nuclear compartment. ${ }^{5}$ However, this experimental setup does not allow for the discrimination between the possible p17 and p19 caspase-3 subunits. To further characterize and reveal the subcellular localization of those caspase-3 subunits in activated microglia, immunoblot analysis for cleaved caspase-3 was performed on both nuclear and cytoplasmic subcellular fractions. The p19 subunit was predominantly found to be localized in the cytosol in both the activated and dying microglia. In contrast, the p17 subunit, which was detected in the dying STS-treated microglia, was detected in both the cytoplasmic and nuclear compartments (Figure 1b). Collectively, these data indicate the presence of cytoplasmic active caspase-3 in pro-inflammatory-activated microglia, in contrast to whole-cell (cytoplasm/nucleus) caspase-3 activation in the dying cells. These conclusions are further supported by our previous finding that $\operatorname{PKC} \delta$, a cytoplasmic caspase-3 substrate, but not poly(ADP-ribose) polymerase (PARP-1) a nuclear caspase-3 nuclear substrate, is found to be cleaved in microglia cells in response to LPS treatment. ${ }^{5}$

Pro-inflammatory activation of microglia is associated with induction of caspase-3-interacting protein clAP2. Thereafter, we aimed at elucidating the molecular mechanism that controls the restricted caspase- 3 processing in microglia cells and thereby prevents their death and contributes to their activation towards a pro-inflammatory phenotype. Given the devastating consequences of caspase 
activation, cells have evolved mechanisms to regulate caspase activity. Inhibitors of apoptosis protein (IAPS) constitute an important class of apoptosis regulators functioning at the level of caspase activity. ${ }^{13,14}$ They recognize, through baculovirus IAP repeat (BIR) domains, a specific sequence found on caspases called the IAP-binding motif (IBM). The IBM is found on the N-terminal of the p19 peptide but is absent (through autocatalytic cleavage) from the p17 peptide. X-linked IAP (XIAP), the prototypical IAP in mammals, inhibits apoptosis largely through direct inhibition of the initiator caspase- 9 and the effector caspase- 3 and caspase-7. Two additional IAP family members, clAP1 and clAP2, were once thought to also inhibit caspases, but more recent studies suggest that these IAPs bind to effector caspases but do not inhibit their proteolytic activities. ${ }^{15-17}$ It is very attractive to envisage that upregulation of IAPs during the caspase-mediated microglia activation is responsible for holding back caspase-3 in terms of activity and subcellular localization and therefore would be a key molecular event in preventing the cell death. Therefore, the mRNA expression levels of XIAP, clAP1 and clAP2 in microglia cells after LPS treatment was analyzed. Remarkably, only clAP2 mRNA expression levels were found to be increased in microglia up to $6 \mathrm{~h}$ post LPS treatment (Figure 2a). Interestingly, clAP1 mRNA expression levels were found to be reduced in LPStreated microglia cells. Immunoblot analysis confirmed the increase in clAP2 expression at protein levels in a timedependent manner upon microglia activation in response to LPS treatment (Figures $2 \mathrm{~b}$ and $\mathrm{c}$ ). Worth a notice, LPS treatment has been reported to induce clAP2 expression in macrophages. ${ }^{18}$ In addition, clAP2-deficient mice exhibit profound resistance to LPS-induced sepsis. ${ }^{19}$ Caspase-3clAP2 interaction has previously been reported in various cellular model systems. ${ }^{16,20,21}$ Further, protein-protein interaction between caspase-3 and clAP2 was confirmed in microglia using an in situ proximity ligation assay (PLA) (Figures $3 \mathrm{a}$ and $\mathrm{b}$ ). The predominant cytoplasmic localization of clAP2 was confirmed by immunoblot analysis performed on both the nuclear and cytoplasmic subcellular fractions (Figure 3c).

cIAP2 controls the conversion of p19 into p17 caspase-3 subunit in microglia. As previously mentioned, the IBM is found in the short prodomain of caspase-3 and is present in the p19 peptide but is absent from the p17 peptide. Interestingly, the processing of the caspase-3 p19 fragment to the p17 fragment has been suggested to be controlled by clAP2. ${ }^{22}$ To investigate the functional role of the caspase-3clAP2 association, we next determined the effect of clAP2 silencing using small interfering RNA (siRNA) (Figures 3d and e) on the processing of caspase-3 upon LPS-induced BV2 microglia activation. Immunoprecipitation using the cleaved caspase-3 Asp175 antibody, which recognized p17 and p19 subunit, was used to isolate and concentrate caspase-3 subunits. Subsequent immunoblot analysis using the same antibody revealed that overall cleaved caspase-3 levels were increased and the generation of the caspase- 3 p17 fragment in LPS-treated microglia was increased by 1.5fold in cells transfected with a clAP2 siRNA as compared with control siRNA (Figures $3 d$ and e). DEVD-ase activity assay also revealed that clAP2 siRNA positively affected caspase3-like activity in LPS-treated microglia cells (Figure 3f).

clAP2 silencing promotes cell death and hinders microglia activation upon pro-inflammatory stimulus. The levels of clAP2 can help to determine the threshold of caspase-3 activity that a cell can withstand before succumbing to the irreversible caspase cascade leading to apoptosis. Moreover, the control of caspase-3 p19 to p17 subunit conversion by clAP2 can regulate the subcellular localization of active caspase-3, that is, p19/p12 complexes being cytoplasmic and p17/p12 complexes exhibiting the ability to relocate into the nucleus. The selective localization of active caspase-3 complexes can in turn regulate their ability to

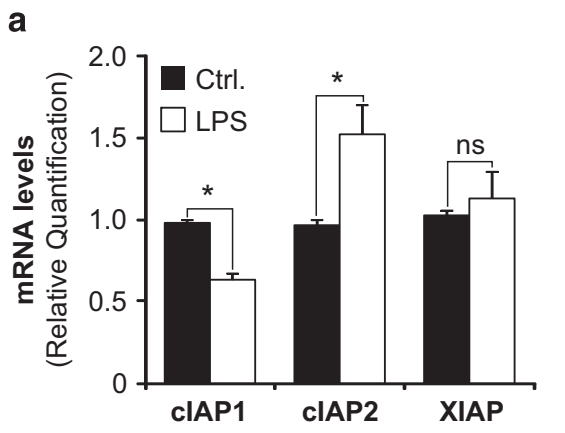

b
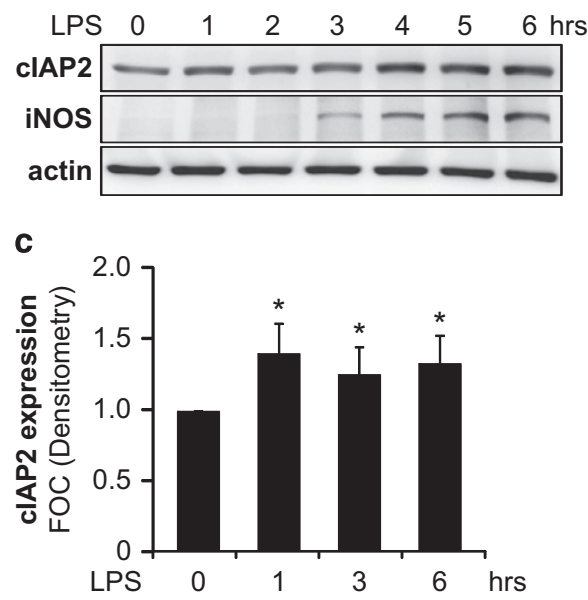

Figure 2 Levels of CIAP2 increase in BV2 microglia cells after LPS treatment. (a) mRNA levels of cIAP1, CIAP2 and XIAP were assessed by qPCR in untreated and $6 \mathrm{~h}$ LPStreated BV2 microglia cells. (b) LPS-induced cIAP2 protein expression was analyzed by immunobloting for the indicated time points. iNOS expression was used as a marker for microglial pro-inflammatory activation and $\beta$-actin used as standard for equal loading of protein. (c) Quantification of clAP2 protein expression is depicted for LPS-treated BV2 cells. Data are expressed as mean \pm S.E.M.; $n=3 ;{ }^{*} P<0.05$ 
a

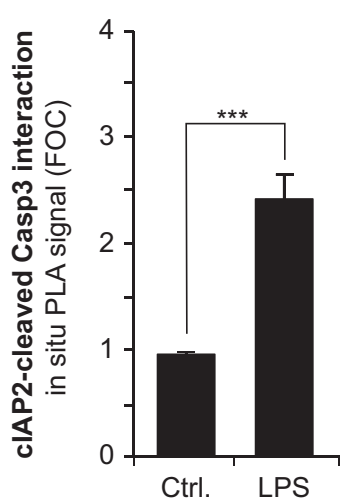

b

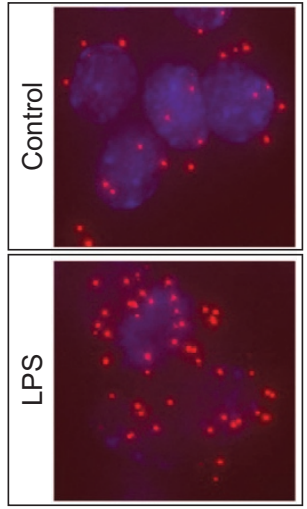

d
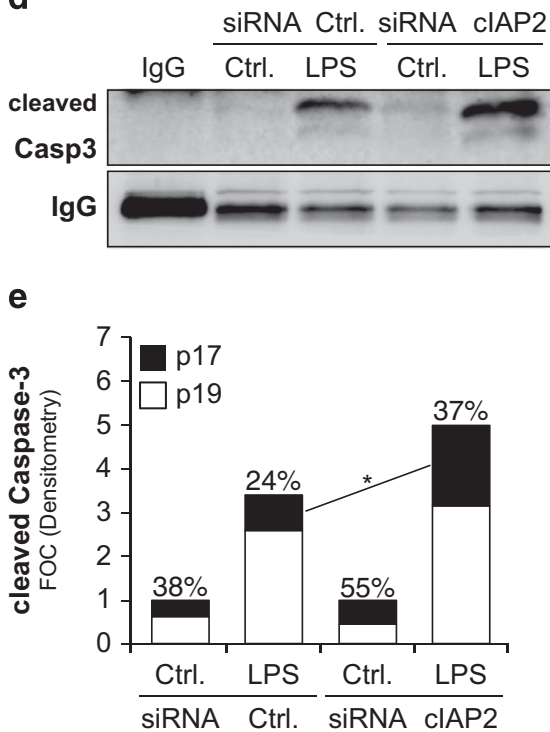

C

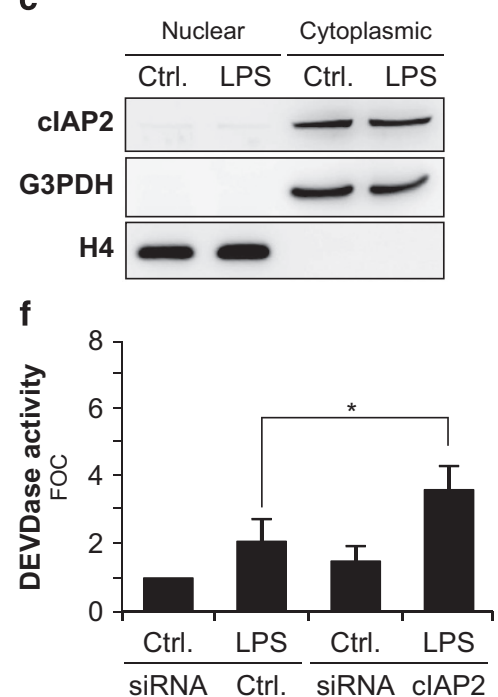

g

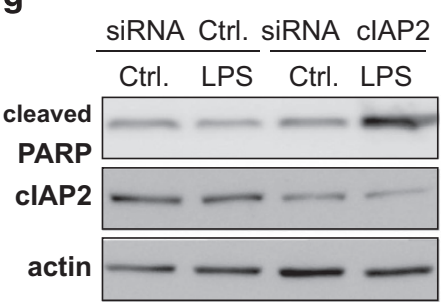

h

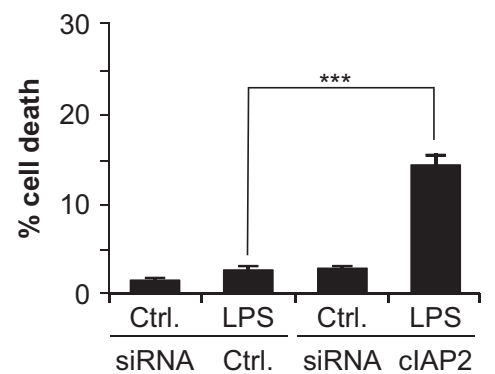

Figure 3 clAP2 interaction with cleaved caspase-3 regulates the conversion of p19 into p17 caspase-3 subunit, its enzymatic activity and apoptosis in microglia. (a) Quantification of in situ PLA demonstrating protein interactions between cleaved caspase-3 Asp175 and cIAP2 occurring in LPS-treated BV2 microglia cells as compared with untreated cells. Data are presented as mean \pm S.E.M. of fluorescent dots/cell; $n=3 ;{ }^{* \star *} P<0.001$. (b) Representative images of PLA in control and LPS-treated cells are depicted. Interactions between cIAP2 and cleaved caspase-3 Asp175 are shown as red dots. (c) Subcellular fractionation illustrates the cytoplasmic localization of clAP2. (d) Immunoprecipitation and subsequent immunoblot using an anti-cleaved caspase-3 Asp175 antibody revealed that selective siRNAs knockdown of cIAP2 promotes caspase-3 p19 to p17 subunit conversion in LPS-treated BV2 cells. Non-targeting siRNAs were used as control. (e) Densitometry analysis of immunoblots showing the total levels of cleaved caspase-3 and the 17 to 19 ratio. Graphed values are mean of three independent experiments. Values above each bar represent the percentage of p17 fragment observed. Increased p19 to p17 conversion upon clAP2 silencing was found to be statistically significant, ${ }^{*} P<0.05$ (f) DEVD-ase activity, (g) immunoblot against cleaved PARP, cIAP2 and $\beta$-actin and (h) cell death quantification in untreated or LPS-treated BV2 microglia cells after knockdown with non-targeting or clAP2-targeting siRNAs pool. Data are expressed as mean \pm S.E.M.; $n=3 ; P<0.05,{ }^{* *} P<0.01$

process cytoplasmic substrates (e.g., PKC $\delta$ ) or nuclear substrates (e.g., PARP) and thereby affect different biological processes such as microglia pro-inflammatory activation or apoptotic cell death, respectively ${ }^{5}$ (see scheme in Figure 6). In agreement, increased PARP cleavage was observed in LPS-treated BV2 cells, where clAP2 expression was targeted using an siRNA strategy (Figure $3 \mathrm{~g}$ ). As a result, increased cell death, as monitored by the appearance of fragmented, damaged or condensed nuclei and apoptotic phosphatidylserine (PS) exposure, was observed upon clAP2 silencing in
LPS-activated microglia (Figure $3 \mathrm{~h}$ and supplementary Figure S1a). Following LPS treatment, BV2 microglia cells did show features of activated microglia, such as inducible NO synthase (iNOS) expression and production of cytokine interleukin-1 $\beta$ (IL-1 $\beta$ ) (Figures $4 a$ and e). Remarkably, when we transfected BV2 microglia cells with siRNA targeting clAP2 (Figures $4 a$ and b), LPS treatment failed to induce iNOS mRNA and protein expression (Figures $4 a, c$ and d) and production of IL- $1 \beta$ cytokine (Figure $4 \mathrm{e}$ ) as effectively. Collectively, these data indicated that clAP2 silencing affects 
a

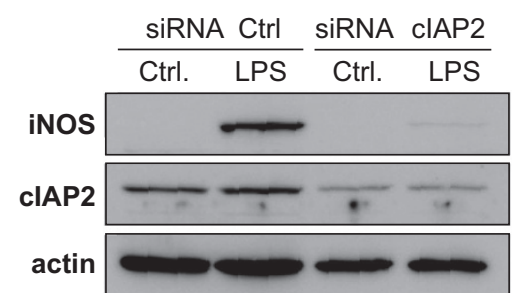

b

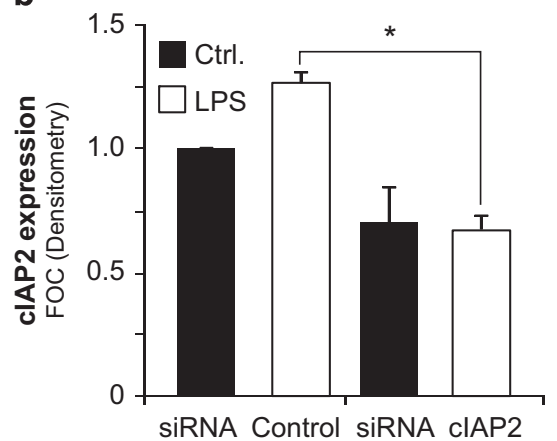

C

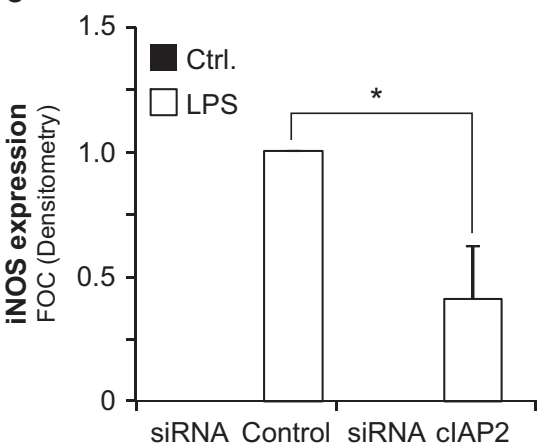

d

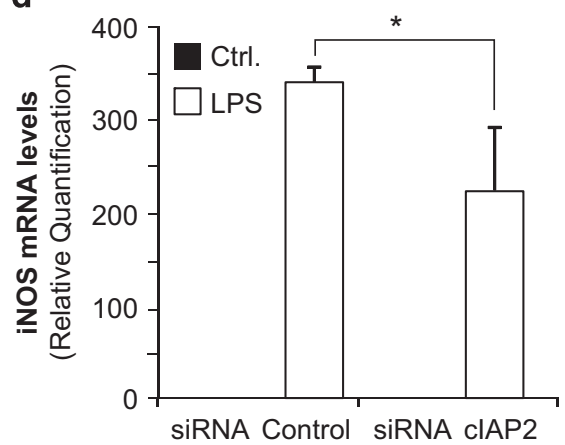

e

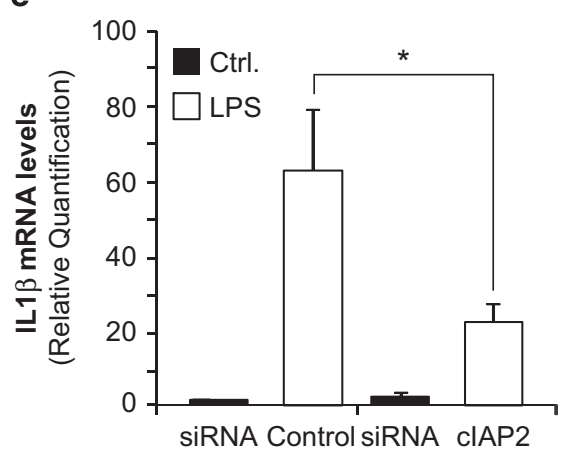

Figure 4 ClAP2 silencing hinders microglia pro-inflammatory activation upon LPS treatment. Knockdown of cIAP2 expression using siRNA prevents LPS-induced expression of iNOS in BV2 microglia cells. (a) Representative immunoblot using anti-iNOS, anti-cIAP2 and anti-actin antibodies. Quantification of (b) clAP2 and (c) iNOS protein expression. (d) iNOS and (e) IL-1 $\beta$ mRNA expression levels were assessed by QPCR in untreated and LPS-treated BV2 microglia cells, transduced with non-targeting control or clAP2 siRNAs. Values are average \pm S.E.M. of three independent experiments

both the microglial cell death and activation under a proinflammatory stimulus like LPS. Thus, disruption of the clAP2 control of microglial caspase-3 activation appears to exhibit pleotropic effects, possibly as a result of the promotion of p19 to p17 caspase-3 conversion, that should result in an increase of the p17/p12 complexes at the expense of the p19/p12 complexes, which regulate cell death and activation, respectively.

SMAC mimetic BV6 enhances caspase-3 activation and promotes cell death of LPS-activated microglia. Mammalian cells possess natural antagonists of IAP-mediated caspase inhibition such as SMAC (second mitochondriaderived activator of caspases) also known as DIABLO (direct inhibitor of apoptosis-binding protein with low isoelectric point). ${ }^{23,24}$ SMAC facilitates activation of apoptotic caspases by releasing these proteases from the inhibitory interaction with IAPs. In the case of XIAP, SMAC binding results in its dissociation from the bound caspases. In contrast, in the case of clAP1/2, SMAC binding triggers autoubiquitination and proteasomal degradation of these molecules. Compounds mimicking the inhibitory effect of SMAC on IAPS, referred as SMAC mimetics, have been developed with the aim to achieve sensitization of cells to apoptotic cell death. BV6, a SMAC mimetic, has been shown to induce rapid proteasomal degradation of clAPs, and thereby abrogate clAPs-mediated inhibition of caspases, and induce cell death. ${ }^{25,26}$ In view of the importance of clAP2 in the regulation of caspase-3 processing upon pro-inflammatory activation of microglia, we decided to analyze the effects of the SMAC mimetic BV6 on BV2 microglia cells. We first selected a concentration of the BV6 compound (i.e., $1 \mu \mathrm{M}$ ), which only induces moderate cell death in microglia, but reduces efficiently cIAP2 protein expression levels in those cells as investigated by fluorescence-activated cell sorting (FACS) analysis (Figure 5a). Furthermore, BV6 treatment was also efficient at counteracting LPS-induced clAP2 expression in microglia. These results are in agreement with previous studies that demonstrated the proteolytic degradation of clAP1/2 protein upon BV6 treatment in various cell 


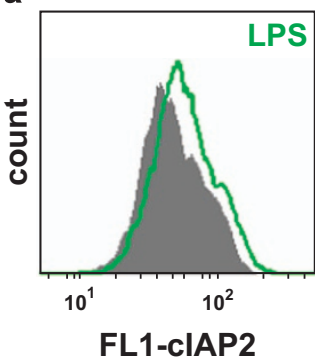

b

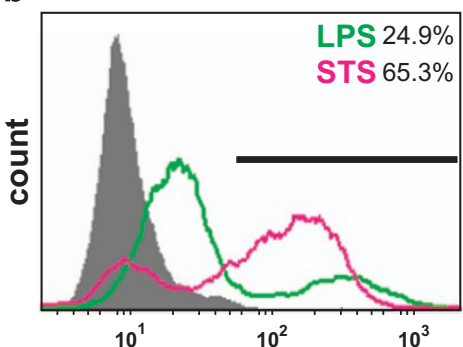

FL1-cleaved Caspase-3

c

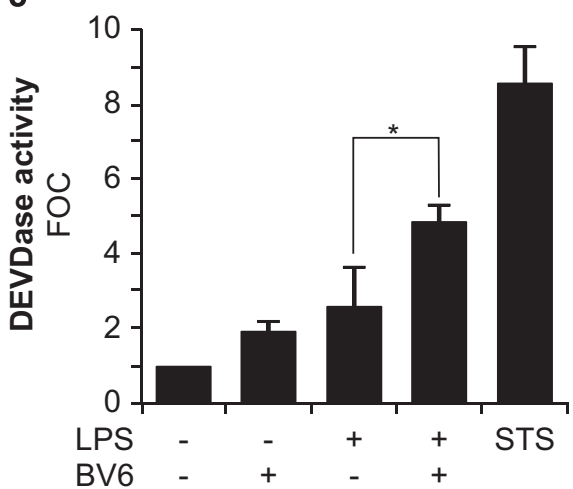

d

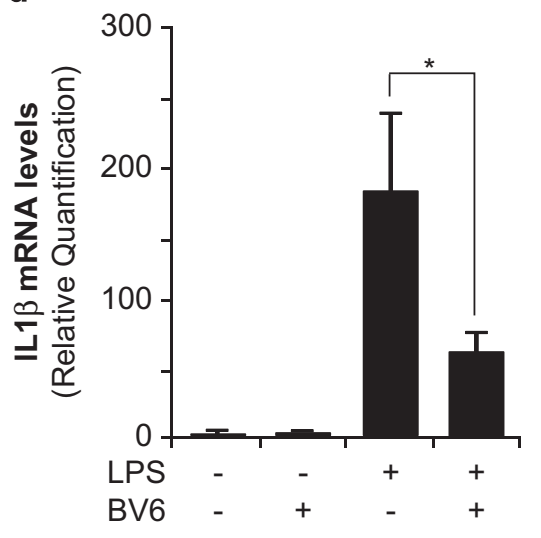

e

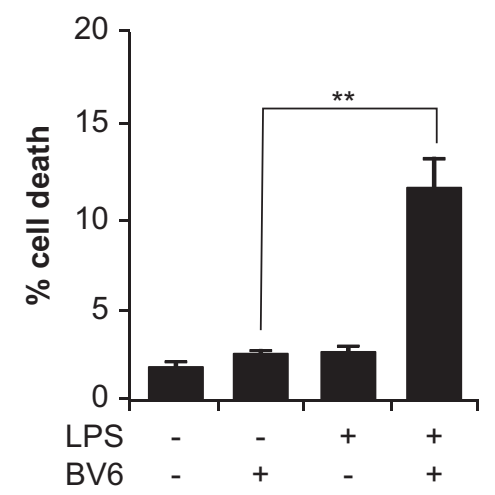

Figure 5 SMAC mimetic enhances caspase-3 activation and promotes cell death of LPS-activated microglia. BV2 microglia cells, pretreated or not with $1 \mu \mathrm{M} \mathrm{BV6}$ compound for $24 \mathrm{~h}$, were subsequently treated with $1 \mu \mathrm{g} / \mathrm{ml}$ LPS for $6 \mathrm{~h}$. STS $(0.1 \mu \mathrm{M})$ for $3 \mathrm{~h}$ was used as cell death stimulus. (a) clAP2 expression was assessed by FACS analysis. Pretreatment with the BV6 SMAC mimetic compound led to (b) increased appearance of cleaved caspase-3 Asp175 as seen by FACS analysis and (c) caspase-3 activity (DEVDase activity), (d) decreased IL-1 $\beta$ mRNA expression and (e) cell death as monitored by the appearance of fragmented, damaged or condensed nuclei in LPS-treated BV2 microglia cells. Data are expressed as mean \pm S.E.M.; $n=3 ;{ }^{*} P<0.05 ;{ }^{* \star} P<0.01$

types including myeloid cells. ${ }^{27}$ Noteworthy, the appearance of cleaved caspase-3 Asp175 and associated DEVD-ase activity were found increased in BV6-treated microglia cells. Moreover, BV6 treatment significantly enhanced these two parameters in LPS-treated microglia cells (Figures $5 b$ and $c$ ). In contrast, decreased LPS-induced IL-1 $\beta$ expression was observed upon exposure to the BV6 compound (Figure $5 \mathrm{~d}$ ). Finally, in agreement with the above described negative impact of clAP2 on caspase-3 processing towards the death-effective p17/p12 complexes, the SMAC mimetic BV6 promoted cell death of LPS-treated microglia (Figure $5 e$ and supplementary Figure S1b and supplementary Figure S2). Altogether, these results demonstrated that the SMAC mimetic BV6 is indeed able to suppress the inhibitory effect of clAP2 on full maturation of caspase-3 complexes and thereby promote microglial cell death (Figure 6).

\section{Discussion}

There is compelling evidence that within the caspase family of cysteinyl aspartate-specific proteases, caspase-3 can perform as a key executor of the apoptotic cell death program. ${ }^{28}$ Activated caspase-3 leads, for example, to the processing of PARP, a caspase-3 nuclear substrate, and thereby contributes to demise of the cell. Nevertheless, increasing number of studies likewise call attention to the non-apoptotic functions for this so-called apoptotic caspase, including in the neuronal cell population. For instance, regulation of synaptic plasticity and function has been shown to be associated with caspase-3 activation. ${ }^{29}$ The non-apoptotic functions of caspase-3 in the brain are not limited to the neuronal population. Indeed, we recently uncovered an unexpected role for caspase-3 in the process of microglia pro-inflammatory activation. ${ }^{5}$ Activation of microglia and inflammation-mediated neurotoxicity are 


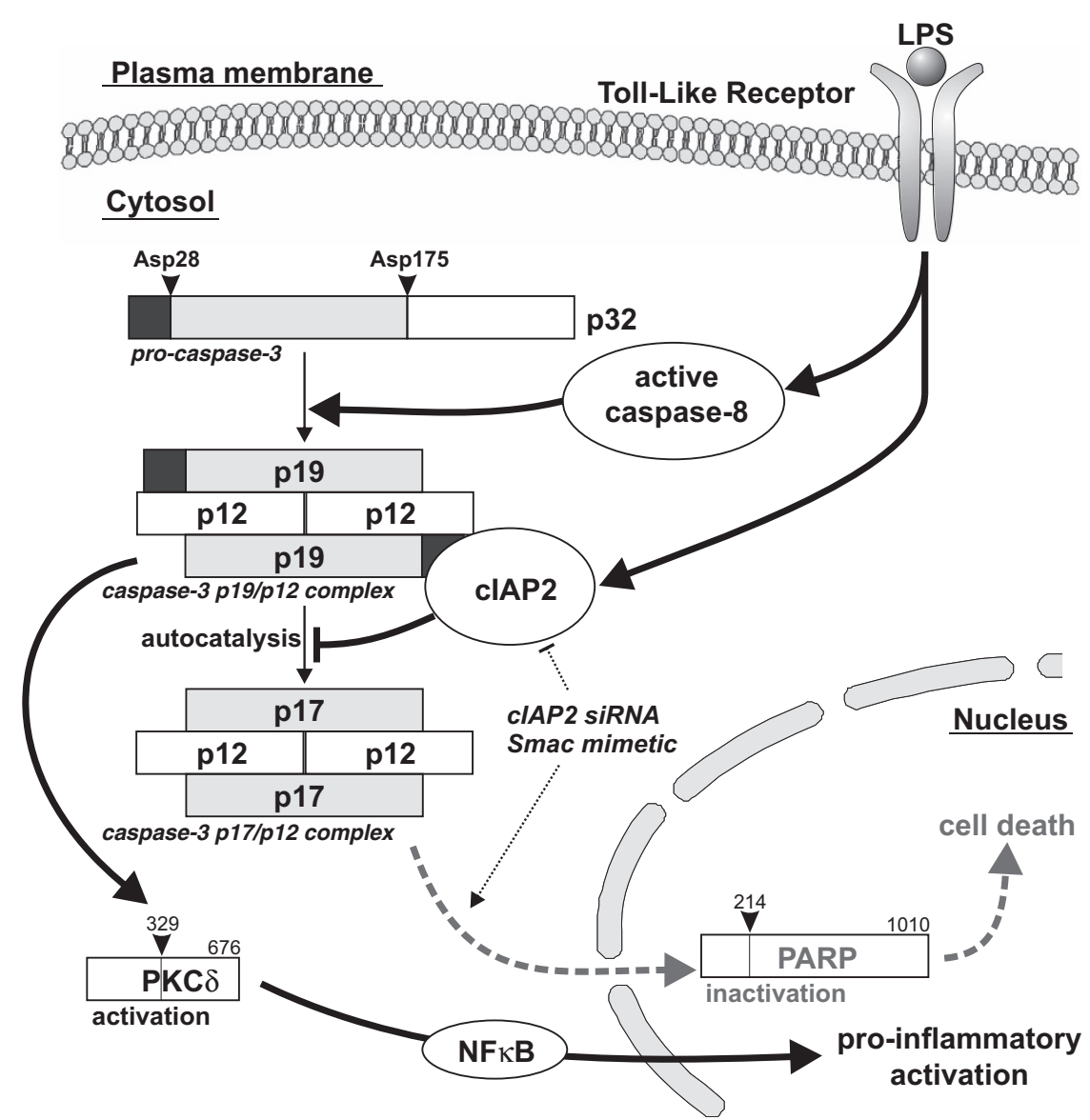

Figure 6 Scheme illustrating the effect of cIAP2 on the caspase-3 activation steps and consequently biological functions. Pro-caspase-3 is cleaved by upstream caspases, such as active caspases-8, at Asp175 to generate intermediate, yet still active, p19/p12 complexes. Thereafter, autocatalytic processing at residue Asp28 and removal of the short prodomain from the p19 peptides, generate p17/p12 complexes that form the fully mature form of the enzyme and translocate to the nucleus. In turn, p19/p12 and p17/p12 complexes can cleave substrates in the cytoplasmic or cytoplasmic/nuclear cell compartment, respectively, and thereby regulate pro-inflammatory activation or apoptotic cell death of the microglia cells. Upon pro-inflammatory stimulation, upregulated cIAP2 binds to caspase-3 prodomain and prevents the p19 to p17 caspase-3 subunit conversion

believed to have important roles in the pathogenesis of several neurodegenerative disorders. We showed that orderly activation of caspase-8 and caspase-3 regulates microglia activation through a $\operatorname{PKC} \delta$-dependent pathway. Thus, caspase-3 activation and subsequent proteolytic activation or inactivation of cytoplasmic (e.g., PKC $\delta$ ) or nuclear (e.g., PARP) caspase-3 downstream targets can lead to rather contrasting effects, microglia pro-inflammatory activation or apoptotic cell death. What restricts caspase- 3 activation in terms of subcellular localization and intensity might provide an answer to the conflicting functions of caspase- 3 in microglia cells. We uncovered that different profiles of caspase-3 processing can be observed in activated versus dying microglia cells, that is, the $p 19$ caspase- 3 subunit seems to occur predominately in activated microglia, whereas the $\mathrm{p} 17$ caspase- 3 subunit arises mostly during the apoptosis. Interestingly, the processing of the cytosolic caspase-3 p19 fragment to the p17 fragment, which has the ability to relocate into the nuclear compartment, has been suggested to be controlled by clAP2. ${ }^{22}$ We and others report that clAP2 protein expression is upregulated in microglia and macrophages upon treatment with a proinflammogen such as LPS. ${ }^{19}$ In addition, clAP2-deficient mice have been reported to exhibit profound resistance to LPS-induced sepsis, specifically because of an attenuated inflammatory response. ${ }^{19}$ We demonstrate that counteracting the repressive effect of clAP2 on caspase-3 activation, using SiRNA targeting clAP2 itself or a SMAC mimetic such as the BV6 compound, reduced the pro-inflammatory activation of microglia cells and promoted their death. On the basis of our findings, we propose that the different caspase-3 functions in microglia, and potentially other cell types, resides in the active caspase-3 complexes formed (Figure 6). Cytoplasmic caspase-3 p19/p12 complexes would be responsible for the pro-inflammatory activation of microglia, whereas the nuclear caspase-3 p17/p12 complexes would promote apoptosis in microglia. Collectively, our data reveal that clAP2 may work as a molecular switch for the decision between pro-inflammatory activation and apoptosis in microglia. These results also could indicate clAP2 as a possible therapeutic target to modulate microglia pro-inflammatory activation and associated neurotoxicity observed, for example, in neurodegenerative disorders. SMAC mimetics, which oppose the effect of clAP2, would also be seen as potential candidate drugs. In fact, SMAC mimetics, alone or in association, are already considered as a new class of targeted drugs for the treatment of solid tumors and hematologic cancers with ongoing clinical 
trials (www.clinicaltrials.gov). ${ }^{30}$ Our results further illustrate the potential impact of SMAC mimetics on microgliaassociated brain diseases, comporting a neuroinflammatory component, such as neurodegenerative disorders, brain trauma or even stroke. However, the benefit of killing microglia cells, instead of suffering their pro-inflammatory activation remains to be proven and would require further investigations. In summary, we show that the clAP2 is involved in regulating caspase-3 activation and thereby functions in microglia. We conclude that regulation of the conversion of caspase-3 p19 subunit to $\mathrm{p} 17$ subunit in those cells can also be used to modulate microglial biological functions.

\section{Materials and Methods \\ Cell culture, transfection and treatments. Murine microglial BV2 cell line was cultured as described. ${ }^{31}$ Cells were maintained in DMEM medium (Gibco BRL, Carlsbad, CA, USA) supplemented with penicillin (100 $\mu \mathrm{g} / \mathrm{ml})$, streptomycin $(100 \mu \mathrm{g} / \mathrm{ml})$ and $10 \%$ fetal calf serum (FCS), reduced to $5 \%$ FCS while performing the experiments. Transfection of BV2 cells was carried out using Lipofectamine 2000 (Invitrogen, Carlsbad, CA, USA) following the manufacturer's recommendation. Predesigned and tested ON-TARGETplus SMARTpool siRNA against mouse BIRC3 (NM-007464; \#L-062425-00-0005) and non-targeted sequence (\#D-001810- 01-05) were purchased from Thermo Scientific (Walthman, MA, USA). Cells were treated with $1 \mu \mathrm{g} / \mathrm{ml}$ LPS (from Escherichia coli, serotype 026:B6; Sigma-Aldrich, St. Louis, MO, USA) or $0.1 \mu \mathrm{M}$ STS (Sigma-Aldrich) for the indicated period of time. BV6, pan-IAP antagonist bivalent compound (used at $1 \mu \mathrm{M}$ ), was generously provided by Genentech, Inc. (South San Francisco, CA, USA). ${ }^{25}$}

Protein extracts, immunoprecipitation and immunoblotting. Cytoplasmic and nuclear protein fractions were extracted in subcellular fractionation buffer (150 mM NaCl, $50 \mathrm{mM}$ HEPES pH 7.4, 0.5\% NP40, $2 \mathrm{mM}$ PMSF and Protease Inhibitor Cockail) alternatively as reported by Joseph et al. ${ }^{32}$ Total protein extracts were made directly in Laemmli buffer. For immunoprecipitation, cells were lysed in an IP lysis buffer $(20 \mathrm{mM}$ Tris- $\mathrm{HCl}$ pH 7.5, $140 \mathrm{mM} \mathrm{NaCl}, 1 \%$ Triton-X100, $2 \mathrm{mM}$ EDTA, $1 \mathrm{mM}$ PMSF, $10 \%$ glycerol and Protease Inhibitor Cocktail) for $15 \mathrm{~min}$ before sonication. Protein G Sepharose (GE Healthcare, Uppsala, Sweden) precleared total protein extracts were incubated with the cleaved caspase-3 (Asp175) rabbit polyclonal antibody in IP lysis buffer overnight at $4{ }^{\circ} \mathrm{C}$. Normal rabbit IgG (\# AB-105-C, R\&D Systems, Minneapolis, MN, USA) was used as control. Immunocomplexes bound to protein $\mathrm{G}$ Sepharose were collected by centrifugation and washed in IP wash buffer (50 mM Tris- $\mathrm{HCl}, \mathrm{pH} 7.5,0.1 \%$ SDS, $1 \% \mathrm{NP} 40$ and $62.5 \mathrm{mM} \mathrm{NaCl}$ ). For immunoblot analysis, protein extracts were resolved on 12 or $15 \%$ SDS-polyacrylamide gel electrophoresis and then blotted onto nitrocellulose membrane. Membranes were blocked in $5 \%$ milk and incubated with antibodies raised against CIAP2 (Santa Cruz Biotechnology, Santa Cruz, CA, USA), cleaved caspase-3 (Asp175) (Cell Signaling, Beverly, MA, USA), cleaved PARP (Cell Signaling), G3PDH (Trevigen, Gaithersburg, MD, USA), iNOS (Santa Cruz Biotechnology), Lamin B (Abcam, Cambridge, MA, USA) or H4 (Active Motif, Carlsbad, CA, USA), overnight at $4{ }^{\circ} \mathrm{C}$, followed by incubation with the appropriate horseradish peroxidase secondary antibody (Pierce, Rockford, IL, USA, 1: 10 000) for $1 \mathrm{~h}$ at room temperature. Immunoblot with anti- $\beta$-actin antibody (Sigma) was used for standardization of protein loading. Bands were visualized by enhanced chemiluminescence (ECL-Plus, Pierce) following the manufacturer's protocol. Densitometry was done using ImageJ software (NIH, USA; http.//imagej.nih.gov/ij).

RNA isolation, cDNA synthesis and qPCR. RNA was isolated from $2 \times 10^{5}$ cells using the total RNA extraction kit (Qiagen, Courtaboeuf, France). cDNA was synthesized from $1 \mu \mathrm{g}$ RNA using Oligo dT, dNTPs and Superscript II (Invitrogen). qPCR was performed using Sybr Green reagents (Applied Biosystems, Waltham, MA, USA) and the following primers (forward; reverse): NOS2 (5'-GTGGT GACAAGCACATTTGG-3'; 5'-AAGGCCAAACACAGCATACC-3), IL-1 $\beta$ (5'-GCTGCT TCCAAACCTTTGAC-3'; 5'-TTCTCCACAGCCACAATGAG-3), CIAP1/BIRC2 (5'-T GATGGTGGCTTGAGATGTTGGGA-3'; 5'-TGAAT CTCATCAACAAACTCCTGACC C-3), cIAP2/BIRC3 (5'-TGTCAGCCAAGTTCAAGCTG-3; 5'-ATCTTCCGAACTTT CTCCAGGG-3), XIAP (5'-CCATGTGTAGTGAAGAAGCCAGAT-3'; 5'-GATCATCA GCCCCTGTGTAGTAG-3) and $\beta$-actin (5'-TTGCTGACAGGATGCAGAAG-3';
5'-TGATCCACATCTGCTGGAAG-3). Results were calculated using delta $\mathrm{Ct}$ method.

Caspase activity assay. Changes in caspase activity in microglia were measured using a luciferase-based assay from Promega (Stockholm, Sweden) known as Caspase-Glo (G890 for caspase-3/7). Equal volumes of cells and kit component were mixed onto a 96-well plate and incubated for $1 \mathrm{~h}$ at room temperature. The plate was analyzed using a luminometer and the value obtained normalized with the number of cells at harvest.

Cell death quantification. After treatment, cells were collected and cytospins were prepared. Subsequently, DNA was stained with Hoechst 33342 $(2 \mu \mathrm{g} / \mathrm{ml}$; Molecular Probes/Invitrogen, Carlsbad, CA, USA). The number of dying cells was measured quantitatively by assessing the percentage of cells with fragmented, damaged or condensed nuclei.

FACS analysis. Appearance of subdiploid-fragmented nuclei derived from apoptotic cells were analyzed after DNA staining with $0.5 \mu \mathrm{g} / \mathrm{ml}$ propidium iodide, in the presence of $2.5 \mu \mathrm{g} / \mathrm{ml}$ RNase A in PBS, pH 7.4. Cleaved caspase-3 or clAP2 expression was quantified in $0.25 \%$ paraformaldehyde-fixed cells following described protocol. ${ }^{5}$ Alexa Fluor 488-conjugated secondary antibodies (Molecular Probes/Invitrogen) were used for detection in the FL1 channel. For determination of the external exposition of PS residues, staining with Annexin-V-FITC and propidium iodide was used according to the manufacturer's instructions (\#V13242, FITC Annexin V/Dead Cell Apoptosis kit, Invitrogen). Data are depicted in logarithmic scales. Analyses were carried out on a FACScalibur flow cytometer equipped with Cell Quest software (Becton Dickinson, Franklin Lakes, NJ, USA).

In situ PLA. Cells were seeded on coverslips and treated as indicated. After treatment, cells were fixed with $4 \%$ paraformaldehyde in PBS. Protein-protein interactions between cIAP2 and cleaved caspase- 3 in fixed cells were detected using the Duolink II in situ PLA from Olink Bioscience (Uppsala, Sweden). PLA was performed in a humidity chamber. After incubation with the blocking buffer (3\% BSA and $0.3 \%$ Triton-X in PBS), cells were incubated with the primary antibodies mouse anti-cIAP2 (1:5000, R\&D Systems) and rabbit anti-cleaved caspase-3 $(1: 400$, Cell Signaling) antibodies, in the antibody diluent medium overnight at $4^{\circ} \mathrm{C}$. Cells were washed with supplied buffer $A$ and incubated for $1 \mathrm{~h}$ in a humidity chamber at $37^{\circ} \mathrm{C}$ with PLA probes detecting mouse or rabbit antibodies (Duolink II PLA probe antirabbit plus and Duolink II PLA probe anti-mouse minus diluted in the antibody diluent to a concentration of $1: 5$ ). After washing with buffer $A$, cells were incubated for $30 \mathrm{~min}$ at $37^{\circ} \mathrm{C}$ with the ligation solution (Duolink II Ligation stock $1: 5$ and Duolink II Ligase $1: 40$ ). If the two protein targets are in close proximity, a template is formed for amplification. Detection of the amplified probe was done with the Duolink II Detection Reagents Red Kit. After repeated washing at room temperature with wash buffer $B$, coverslips were mounted onto slides using mounting medium containing DAPI and samples were observed using an epifluorescence microscope. Protein-protein interaction was measured as the number of fluorescent dots/cell analyzed with Duolink Image tool.

Statistical analysis. Statistical analysis was performed using Student's $t$-test. $P<0.05$ was considered as statistically significant.

\section{Conflict of Interest}

The authors declare no conflict of interest.

Acknowledgements. We thank Drs S Ceccatelli (Karolinska Institutet) and D Vucic (Genentech, Inc.) for providing us with the BV2 cell line and the BV6 compound, respectively. JR is supported by a fellowship from the Karolinska Institutet Foundations and MAB is supported by a postdoctoral fellowship from the Swedish Research Council. This work was supported by the Michael J Fox Foundation for Parkinson's Research, the Parkinson Foundation in Sweden, the Swedish Brain Foundation, the Swedish Cancer Society, the Swedish Childhood Cancer Foundation and the Swedish Research Council.

1. Saijo K, Glass CK. Microglial cell origin and phenotypes in health and disease. Nat Rev Immunol 2011; 11: 775-787. 
2. Block ML, Zecca L, Hong JS. Microglia-mediated neurotoxicity: uncovering the molecular mechanisms. Nat Rev Neurosci 2007; 8: 57-69.

3. Venero JL, Burguillos MA, Brundin P, Joseph B. The executioners sing a new song: killer caspases activate microglia. Cell Death Differ 2011; 18: 1679-1691.

4. McGeer PL, Itagaki S, Boyes BE, McGeer EG. Reactive microglia are positive for HLA-DR in the substantia nigra of Parkinson's and Alzheimer's disease brains. Neurology 1988; 38: 1285-1291.

5. Burguillos MA, Deierborg T, Kavanagh E, Persson A, Hajji N, Garcia-Quintanilla A et al. Caspase signalling controls microglia activation and neurotoxicity. Nature 2011; 472 319-324.

6. Joseph B, Ekedahl J, Lewensohn R, Marchetti P, Formstecher P, Zhivotovsky B. Defective caspase-3 relocalization in non-small cell lung carcinoma. Oncogene 2001; 20 2877-2888

7. Han Z, Hendrickson EA, Bremner TA, Wyche JH. A sequential two-step mechanism for the production of the mature p17:p12 form of caspase-3 in vitro. J Biol Chem 1997; 272 13432-13436.

8. Castano A, Herrera AJ, Cano J, Machado A. Lipopolysaccharide intranigral injection induces inflammatory reaction and damage in nigrostriatal dopaminergic system. $J$ Neurochem 1998; 70: 1584-1592.

9. Saijo K, Winner B, Carson CT, Collier JG, Boyer L, Rosenfeld MG et al. A Nurr1/ COREST pathway in microglia and astrocytes protects dopaminergic neurons from inflammation-induced death. Cell 2009; 137: 47-59.

10. Kamada S, Kikkawa U, Tsujimoto Y, Hunter T. Nuclear translocation of caspase-3 is dependent on its proteolytic activation and recognition of a substrate-like protein(s). J Biol Chem 2005; 280: 857-860.

11. Luo M, Lu Z, Sun H, Yuan K, Zhang Q, Meng $S$ et al. Nuclear entry of active caspase- 3 is facilitated by its $\mathrm{p3}$-recognition-based specific cleavage activity. Cell Res 2010 20: $211-222$.

12. Voss OH, Batra S, Kolattukudy SJ, Gonzalez-Mejia ME, Smith JB, Doseff Al. Binding of caspase-3 prodomain to heat shock protein 27 regulates monocyte apoptosis by inhibiting caspase-3 proteolytic activation. J Biol Chem 2007; 282: 25088-25099.

13. Silke J, Meier P. Inhibitor of apoptosis (IAP) proteins-modulators of cell death and inflammation. Cold Spring Harb Perspect Biol 2013; 5.

14. Gyrd-Hansen M, Meier P. IAPs: from caspase inhibitors to modulators of NF-kappaB, inflammation and cancer. Nat Rev Cancer 2010; 10: 561-574.

15. Tenev T, Zachariou A, Wilson R, Ditzel M, Meier P. IAPs are functionally nonequivalent and regulate effector caspases through distinct mechanisms. Nat Cell Bio 2005; 7: 70-77.

16. Eckelman BP, Salvesen GS. The human anti-apoptotic proteins CIAP1 and cIAP2 bind but do not inhibit caspases. J Biol Chem 2006; 281: 3254-3260.

17. Burke SP, Smith L, Smith JB. clAP1 cooperatively inhibits procaspase-3 activation by the caspase-9 apoptosome. J Biol Chem 2010; 285: 30061-30068.

18. Cui X, Imaizumi T, Yoshida H, Tanji K, Matsumiya T, Satoh K. Lipopolysaccharide induces the expression of cellular inhibitor of apoptosis protein-2 in human macrophages. Biochim Biophys Acta 2000; 1524: 178-182.

19. Conte D, Holcik M, Lefebvre CA, Lacasse E, Picketts DJ, Wright KE et al. Inhibitor of apoptosis protein clAP2 is essential for lipopolysaccharide-induced macrophage survival. Mol Cell Biol 2006; 26: 699-708.
20. Huang $\mathrm{H}$, Joazeiro $\mathrm{CA}$, Bonfoco $\mathrm{E}$, Kamada S, Leverson JD, Hunter $\mathrm{T}$. The inhibitor of apoptosis, clAP2, functions as a ubiquitin-protein ligase and promotes in vitro monoubiquitination of caspases 3 and 7. J Biol Chem 2000; 275: 26661-26664.

21. Durkop H, Hirsch B, Hahn C, Stein H. clAP2 is highly expressed in Hodgkin-Reed-Sternberg cells and inhibits apoptosis by interfering with constitutively active caspase-3. $J \mathrm{Mol}$ Med (Berl) 2006; 84: 132-141.

22. Rajalingam K, Sharma M, Paland N, Hurwitz R, Thieck O, Oswald M et al. IAP-IAP complexes required for apoptosis resistance of $\mathrm{C}$. trachomatis-infected cells. PLOS Pathog 2006; 2: e114.

23. Du C, Fang M, Li Y, Li L, Wang X. Smac, a mitochondrial protein that promotes cytochrome c-dependent caspase activation by eliminating IAP inhibition. Cell 2000; 102: 33-42.

24. Verhagen AM, Ekert PG, Pakusch M, Silke J, Connolly LM, Reid GE et al. Identification of DIABLO, a mammalian protein that promotes apoptosis by binding to and antagonizing IAP proteins. Cell 2000; 102: 43-53.

25. Varfolomeev E, Blankenship JW, Wayson SM, Fedorova AV, Kayagaki N, Garg P et al. IAP antagonists induce autoubiquitination of c-IAPs, NF-kappaB activation, and TNFalpha-dependent apoptosis. Cell 2007; 131: 669-681.

26. Vince JE, Wong WW, Khan N, Feltham R, Chau D, Ahmed AU et al. IAP antagonists target CIAP1 to induce TNFalpha-dependent apoptosis. Cell 2007; 131: 682-693.

27. Muller-Sienerth N, Dietz L, Holtz P, Kapp M, Grigoleit GU, Schmuck C et al. SMAC mimetic BV6 induces cell death in monocytes and maturation of monocyte-derived dendritic cells. PloS One 2011; 6: e21556.

28. Nicholson DW, Ali A, Thornberry NA, Vaillancourt JP, Ding CK, Gallant M et al. Identification and inhibition of the ICE/CED-3 protease necessary for mammalian apoptosis. Nature 1995; 376: 37-43.

29. D'Amelio M, Cavallucci V, Cecconi F. Neuronal caspase-3 signaling: not only cell death. Cell Death Differ 2010; 17: 1104-1114.

30. Beug ST, Tang VA, Lacasse EC, Cheung HH, Beauregard CE, Brun J et al. Smac mimetics and innate immune stimuli synergize to promote tumor death. Nat Biotechnol 2014; 32 : 182-190.

31. Bocchini V, Mazzolla R, Barluzzi R, Blasi E, Sick P, Kettenmann H. An immortalized cell line expresses properties of activated microglial cells. J Neurosci Res 1992; 31: 616-621.

32. Joseph B, Wallen-Mackenzie A, Benoit G, Murata T, Joodmardi E, Okret S et al. p57(Kip2) cooperates with Nurr1 in developing dopamine cells. Proc Natl Acad Sci USA 2003; 100: $15619-15624$

(i) Cell Death and Disease is an open-access journal published by Nature Publishing Group. This work is licensed under a Creative Commons Attribution 4.0 International Licence. The images or other third party material in this article are included in the article's Creative Commons licence, unless indicated otherwise in the credit line; if the material is not included under the Creative Commons licence, users will need to obtain permission from the licence holder to reproduce the material. To view a copy of this licence, visit http://creativecommons.org/licenses/by/4.0 